\title{
Exploring 3D Playblast-To-2D Animation Rotoscoping Techniques
}

\author{
ASHRUL SYARIFUDDIN* \& TERRY LUCAS* \\ Faculty of Applied and Creative Arts, Universiti Malaysia Sarawak, \\ 94300 Kota Samarahan, Sarawak,Malaysia \\ *Corresponding authors: 21020030@ siswa.unimas.my, lterry@unimas.my
}

\begin{abstract}
Rotoscoping can produce believable 2D animation and movements when the process is done correctly. However, not all live-action footage can be easily rotoscoped due to scenes or fictional designs that are impossible to shoot. To overcome this technical challenge, 3D playblast can be used as footage for an alternative source for rotoscoping. This research aims to explore and evaluate two rotoscoping techniques of transforming 3D playblast into 2D animation. Two different animation software (Adobe Animate CC and Toon Boom Harmony) were used to explore those rotoscoping techniques. The method began by designing a storyboard with dynamic camera angles. Then, animated 3D models were developed based on the storyboard as footage for rotoscoping. Then, those animated 3D models were rotoscoped frame by frame using 'Toon Boon Harmony' and 'Adobe Animated CC'. The rotoscope outputs were evaluated for their effectiveness and weaknesses. Findings show that rotoscoping frame by frame is viable for both software with some nuances. Nevertheless, the proposed rotoscope techniques can maintain consistency between frames without losing the proportion of anatomy and style when animating in an extreme and distorted scene.
\end{abstract}

Keywords: animation; 2D animation; 3D playblast; rotoscope; Adobe Animate CC; Toon Boom Harmony

Copyright: This is an open-access article distributed under the terms of the CC-BY-NC-SA (Creative Commons Attribution-Non-Commercial-Share Alike 4.0 International License), which permits unrestricted use, distribution, and reproduction in any medium, for non-commercial purposes, provided the original work of the author(s) is properly cited.

\section{INTRODUCTION}

The origin of the rotoscope can be traced back to 1917 when a studio named Fleischer Studios from New York officially patent their machine 'Rotoscope'. For several years, the machine was a success and created the Out of the Inkwell (1918-1929) series. Hence, a few famous cartoons and characters such as Koko the Clown, Betty Boop, and Popeye became iconic characters from the series (Bratt, 2011). Moreover, the Rotoscope machine is consists of a camera that is mounting behind the desk while the film footage is projecting onto a slate of frosted glass. Next, the animator will trace it onto a paper before retracing it onto a clear animation cell to create the key drawing. As time progresses, the Rotoscope machine begin to evolve towards a new modern look and technique.

Currently, animators can view the footage while animating it. It helps established and identify easier keyframes as well as isolating and separating layers of selected footage. Despite the digital change of the machine, the workflow and concept remain the same (Bratt, 2011). However, the issue arises when specific complex or dynamic scenes are not easily captured using live-action footage. These dynamic scenes can be critical for the animation's narrative, but they may be too costly or dangerous to capture in live-action in which will be used as the footage for the rotoscoping process. Therefore, this study suggests an alternative approach which is by using $3 \mathrm{D}$ playblast instead.

This research aims to explore two approaches of rotoscoping techniques from 3D playblast into 2D animation. The project development starts by creating the rough layout of the animation, such as the scripts, storyboard and animatics. Once the rough outline is acquired, several scenes are selected and turn into 3D playblast, consisting of moving and static models. Moreover, the chosen scene is composed around the element of dynamic: focal lengths, horizontal and vertical film aperture, and camera movement with various angles and speeds. Furthermore, the scene will be exported into the separate subject matter, consist of background and character models, before rotoscoping it in two separate software with a different approaches of rotoscoping. It is hoped that by exploring the rotoscoping from the 3D playblast approach, more dynamic scenes and interesting movements can be created for $2 \mathrm{D}$ animation.

\section{LITERATURE REVIEW}

\section{Element of Dynamic Camera Scenes}

Camera angles can direct the audience to see and affect how they perceive it. Dynamic camera scenes are added to the project to test further the limitation of a fictional scene that is extremely hard to record. One of the 
cinematographer's greatest weapons is positioning the view related to the subject or scenery. As a result, each camera angle focuses on the shot have its own each emphasises and affects the overall mood (Gabe, 2014). Heiderich (2012) provides an excellent explanation about the meaning behind the type of shot used in the filmmaking industry. He states that an extreme long shot is typically used to display large-scale subjects and express how insignificance the character struggles against its surroundings. A long shot can reflect an emotional distance base on the camera distance. The audience would not get as emotionally involved if they were closer, making the character feel detached from their surroundings. Moreover, a medium shot is used to engage with the characters personally, like how we casually have a conversation. An extension from the previous shot, the close-up is more intimate than the medium shot. The character expresses more visible emotions, which can relate when engaging in a direct personal manner. Additionally, an extreme close-up is often used as an amplifier for emotional intensity and expressions.

\section{Modern Rotoscope Technique}

Rotoscoping is an animated technique in which the footage of either a digitised or animated subject is traced over (Jaran, 2015). A few authors have described methods of automatic rotoscoping as an estimation of simultaneous tracking motion over the entire sequence. One of the methods is using Optical flow by tracking the pattern of the apparent movement of the objects in an image (Irani, 2002). Another method is Point Tracking which its purpose is to track a new procedure that can track flexible motion by integrating space-time constraints (Torresani \& Bregler, 2002). Both methods are designed to operate automatically. Furthermore, modern tracking techniques enable the creator to 'artificial intelligently' track multiple scenes without manually rotoscope them. However, it certainly would not work for every scene inside the footage. The problem lies in the elements in the scene, background parts that are similar to the foreground subject; hence, it is covered or cluttered due to its similarity of the object (Blake \& Isard, 1998). Compared with the old industrial rotoscoping tool like Pinnacle Commotion: it uses hand positioning the contour manually for each curve in every frame (Agarwala, Hertzmann, Salesin, \& Seitz, 2004). However, a hybrid concept of manual and automatic tracking also exists, such as the roto-curve; calculated tracking by manually drawing roto-curve (outline) and automatically generating roto-curve for the follow-up frame to follow the deformation of the motion closely with the corresponding roto-curve (Agarwala et al., 2004).

\section{Rotoscoping from 3D Computer-Generated Imagery (CGI)}

Rotoscoping from live footage was common knowledge as the first-ever rotoscoping technique. However, the growing media, especially the $3 \mathrm{D}$, have developed more potential references to trace over. For example, Ufotable Studio is famous for its digital composite by producing fascinating moments using 3D backgrounds, sophisticated VFX, 2D animation, and rotoscoping CGI (Callum May \& Kim Morrissy, 2018). For instance, 2013 demo reel from the digital team composite their work process using the niche rotoscoping CGI technique (Ufotable, 2013). However, rotoscoping live-action material for animation has its limits. For example, the footage of extreme dynamic shots and unusual movement from unreal character concepts are difficult to record via live-action. For instance, Riverman Media (n.d.) created a game entitled 'Pizza vs Skeletons' in which the skeleton's movement is impossible to shoot in actual live-action footage.

\section{Existing Techniques of Rotoscoping using 3D Playblast Animation and Live Action Footage}

Tracing from actual live-action footage was the first rotoscope technique. Several films or animations have risen in popularity throughout the years based on their creative approach using modern rotoscope. For instance, Spot Ceres has created a commercial using a rotoscope by tracing over the footage with cartoon's colour scheme, bold outline, less jittery motion, and surrealism effect, creating this cell shade visual (Spot Ceres, 2012). Moreover, special software like Rotoshop developed by Bob Sabiston interpolated automatically in-between keyframes to visualise dreamlike aesthetic and could be considered a painterly type of animation (Sabiston, 2017). This rotoscope technique visualises the figure through a mesh of shapes and lines that allow a unique aesthetic between live-action and animation (Ruddell, 2012). Swarbrick Richard also has a traditional painterly approach to rotoscoping that focuses on painting and nostalgic sensibility. He first painted individual frames on a computer tablet in Photoshop, animating them in After Effects and then synchronising in original commentary from the original video (Swarbrick, 2021). Other than that, Patterson utilised a rotoscope technique combining live-action with sketches of gesture drawing in A-Ha's music video "Take on Me" (1985) with a 2010 remaster (A-ha, 2010). Rather than stiff outline tracing, the technique focuses more on motion and the feeling of energy (Karpathyova, 2017)

Several similar methods in the past explore the opportunity on rotoscoping 3D animation. For example, the most famous studio that pushes this method is Studio Ufotable. Ufotable's method of rotoscoping 3D sequence into 2D animation significantly improves the scene with over-the-top character fight choreography and extremely complicated tracking shot is part of the studio (Callum May \& Kim Morrissy, 2018; Ufotable, 2013). Another example is rotoscoping an animated 3D model in Blender using the 'Grease Pencil' tool (Coulthard, 2020; Team Miracles, 2020). Moreover, with 3D references, stylised design like the 80's Japanese animation design is possible to create. Rather than character only, sci-fi spaceship or any non-detailed model are also possible to rotoscope by 
using Cinema 4D (Johnson, 2014, 2018).

The noticeable difference between rotoscoping using 3D playblast animation and live-action footage is the realism aspect (Table 1). Playblast is created from the author's understanding of the fundamentals to gain creative freedom. However, real-footage provides the solid groundwork for rotoscope but with more expenses and realistic constraint. The advantages of using live-action footage for rotoscope is its believable motions that captures more minor details that could be found in real life. This is great for a non-complicated shot because it is easier to record. Modern software can automatically rotoscope scenes without the manual labour from the older rotoscope technique. However, the requirement to obtain extreme tracking and perspective footage is based on the project's equipment and budgetary constraint. For now, automatic rotoscoping only applies if the subjects are different from each other and not cluttered together. On the other hand, rotoscoping 3D playblast animation does break the unconventional constraint from live-action footage. In an exchange with more workload, lesser realism, and more technical experience with 3D software animation, the author could pre-visualize their conceptual ideas for their expected scenes. Furthermore, the freedom to be creative with tools such as virtual camera, model, riggings, special effects, and even better workflow could potentially improve the original scene. Unlike live-action footage, the recorded scene within 3D software can be altered anytime and reuse in other projects.

Table 1. Comparison between the technique of rotoscoping 2D animation between live-action footage and 3D playblast.

\begin{tabular}{|c|c|c|}
\hline 3D PLAYBLAST ANIMATION & ASPECTS & LIVE-ACTION FOOTAGE \\
\hline $\begin{array}{l}\text { - Creating imaginable scenarios (pre- } \\
\text { visualisation) } \\
\text { - Freedom to be creative } \\
\text { - No realistic constraint } \\
\text { - Suitable for puppet and classical rotoscope } \\
\text { animation. }\end{array}$ & Advantages & $\begin{array}{l}\text { - Fluid and realistic motion } \\
\text { - Maintain realistic details that difficult to } \\
\text { recreate in imagination } \\
\text { - Modern rotoscoping algorithm that } \\
\text { connects pixel in between keyframe }\end{array}$ \\
\hline $\begin{array}{l}\text { - Required knowledge on the principle of } \\
\text { animation and software } \\
\text { - Higher workload } \\
\text { - Non-realistic details and scenarios to make } \\
\text { it logically realistic }\end{array}$ & Disadvantages & $\begin{array}{l}\text { - Constraint with real-life logic } \\
\text { - Algorithm underperformed in noisy } \\
\text { conditions. }\end{array}$ \\
\hline
\end{tabular}

One of the solutions was to rotoscope in a more modern technique via 3D playblast. Hence, the focus of this research is to analyses various methods of known rotoscope techniques. The following sections will explore the two different rotoscoping methods that refer to the $3 \mathrm{D}$ animation and convert it into $2 \mathrm{D}$ animation. These two divergent techniques are explained in further detail, and the advantages and disadvantages of each rotoscoping approach will be discussed.

\section{METHODS}

The methodology will be focusing on qualitative exploration of 2D rotoscoping techniques based on the acquired 3D playblast animation. Automatic tweening and frame-by-frame techniques will be compared based on their specialised software for each technique. First, the production will follow a standard process of creating animation. Briefly, there are three stages of animation production (Gossman, 2012) and Figure 1 shows the overview of the development process for this study's animation entitled "A Thousand Miles": 


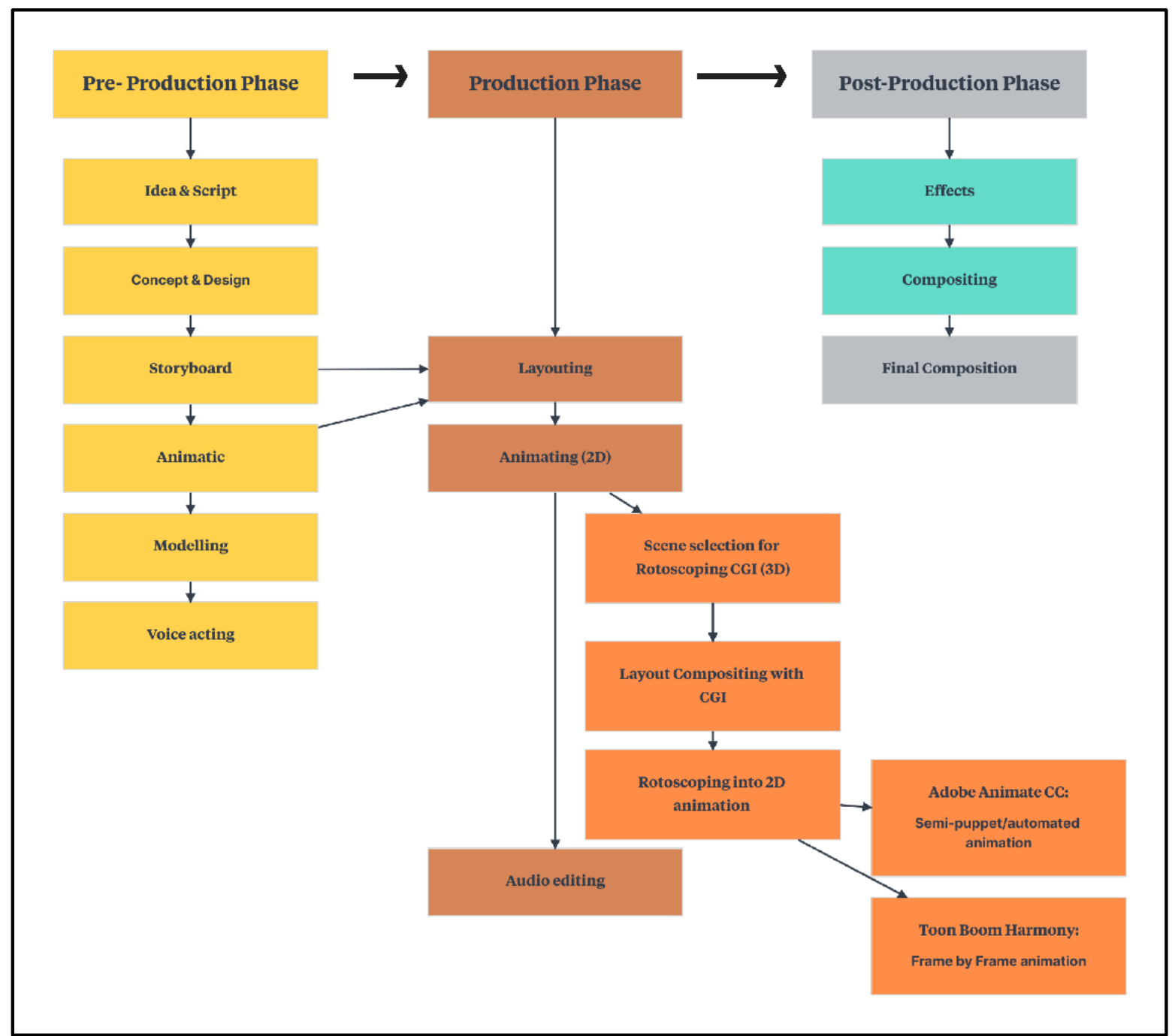

Figure 1. The development process for the short animation "A Thousand Miles."

The animation production "A Thousand Miles" was developed into three phases, starting from the 'Pre-Production' phase. Usually, ideas were translated into a document format known as a script before adjusting its concept and design. Next, the script was represented visually in a storyboard format. Based on the storyboard, individual panels, voice acting, music, and sound effects, are edited together to see how it flows like an animated film. This is referred to as animatic. It served as a blueprint for the final edit. Moreover, a few scenes were selected and modelled into a fully 3D animation layout before the production stage. However, the character model and rigging developed by Uğur Ulvi Yetiskin (2014) were used in the character animation due to the limitations of the researcher equipment.

Next is the 'Production' phase. The first step in this phase is the layout based on the storyboard panels and animatic, including the conceptual design. The following step will be the actual animation: a classical 2D animation approach is by blocking out the character with a keyframe within an environment. In exploring rotoscoping approaches via 3D playblast, several selected dynamic 3D scenes were used as references. Moreover, two mainstream software were used and compared with different animation approaches specialising with each software: (a) Adobe Animate $\mathrm{CC}$ and (b) Toon Boom Harmony. However, high quality rendering of the 3D animation is unnecessary and may clutter the overall view when rotoscoping into $2 \mathrm{D}$ animation.

Finally, finishing touches like special effects, sound, and colour correction were implemented. Compositing and editing start from the earlier animatic stage. The shots went from storyboard to rough animation to final render and ultimately final composite; they were constantly updated to make sure the shots flowed at an appealing pace. This methodology explores the rotoscoping technique of selected software and how they work with 3D playblast as references in developing 2D animation. Adobe Animate CC will be using a semi puppet method because the software contains more automatic tools than the other, efficiently reducing production time and fewer restrictions. Meanwhile, Toon Boom Harmony will be focusing on classical rotoscoping (tracing frame by frame) because the software is one of the closest tools to produce conventional work. 


\section{D Model Creation using Autodesk Maya}

The software that is suitable for the 3D modelling for this project is Autodesk Maya. Autodesk Maya generally enables the user to create models of varying complexity, including a complete product for animation, rendering and shading assets for the projects. Moreover, the product also includes editable movement using the Time Editor and Graph Editor for complex animation via a simple interface. Some benefits of using this software are its customisable and automatable features to complete a task without deep knowledge in programming. Hence, creating a versatile and efficient workflow when completing a project. With that in mind, the scenes started with creating suitable models for the layout of the subject.

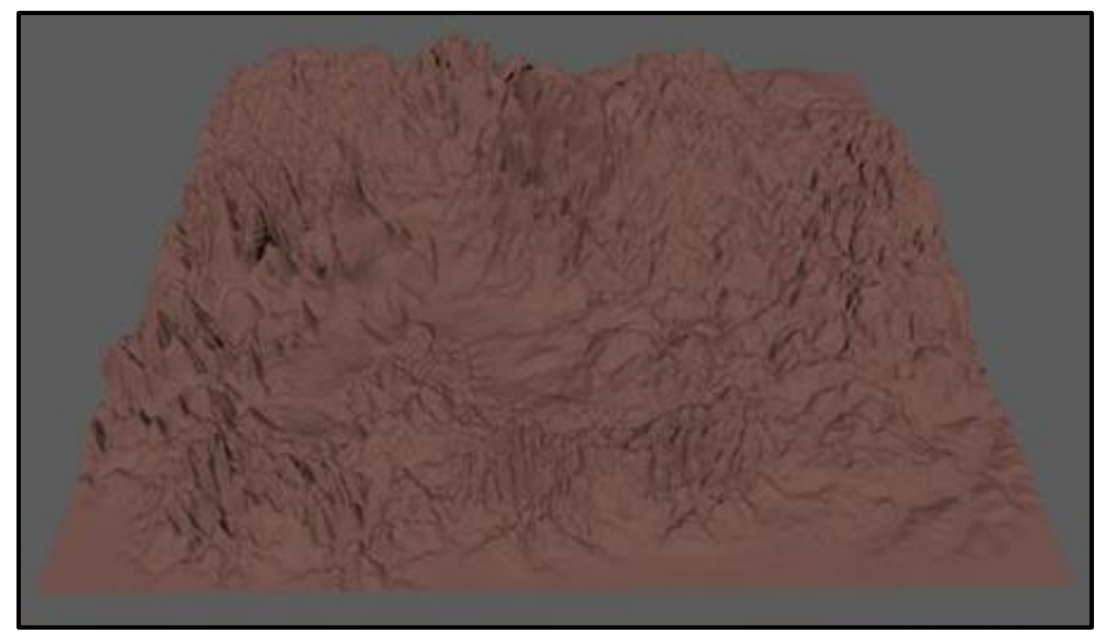

Figure 2. Models of the environment.

The process started with the world-building from the selected scene (refer to Figure 2). Generally, the model is deformed using the sculpt geometry tool to manually sculpt the selected NURBS by pulling or pushing the polygons. The mountains and rock formations can be sculpted using the map height image feature for the rough baseline. Moreover, the pressure-sensitive tablet (Wacom) can conveniently apply the formation height or size that scale accurately with the pen pressure. Hence, an organic landscape with better depth and atmosphere can be built quickly using this feature.

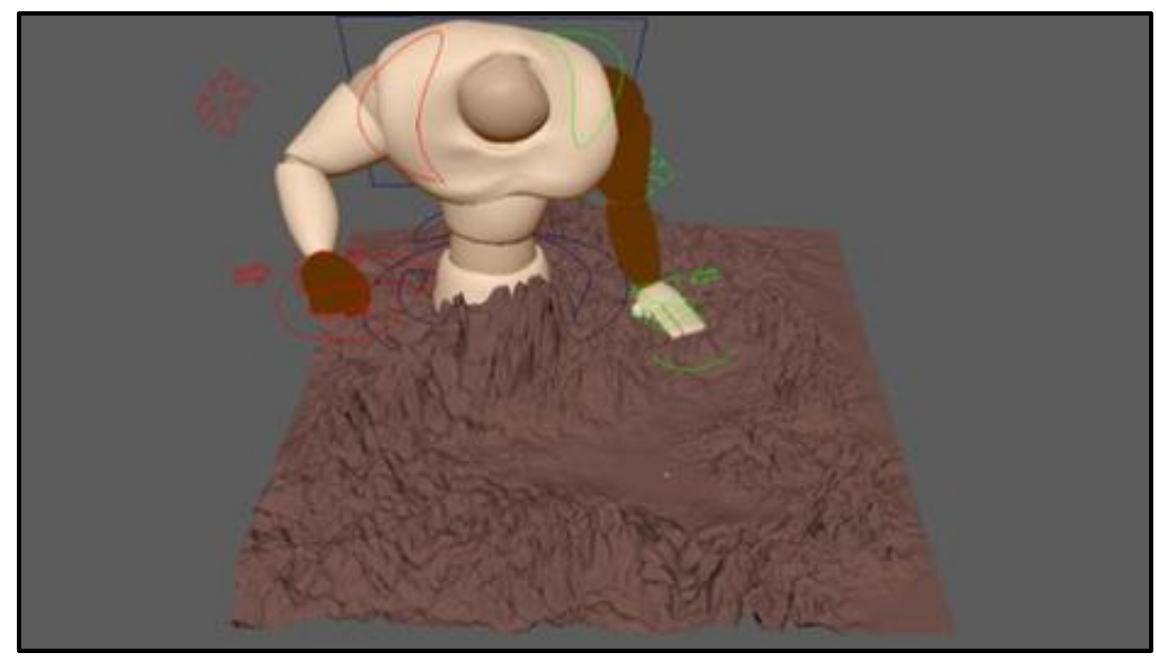

Figure 3. Compositing the subjects.

After creating the environment, the 3D model is imported into a separate scene file. This method ensures the individual file can be recovered when unfortunate situations happen, such as crashing and computing problems during the animation phase. A model from Uğur Ulvi Yetiskin (2014) was selected as the character for the rotoscope. Conceptually, the model is made of simple shapes and character silhouettes. The main objective of this model is to create a proper separation that is easily identified during the rotoscoping process, without adding complicated features such as hair and clothes physic that potentially clutter the overall shapes or silhouette. 
Generally, to strengthen the story point in any scene, each subject within a scene must acquire its reason to exist, and each subject should at least have a connection with one another. In summary, the subject should have a purpose on why it exists in that scenario or scene to give context for the story. For example, Figure 3 shows the main character confronting the monster within the wasteland. In this scene, the rock formation shows a deserted area. The encounter shows suspense while building intensity.

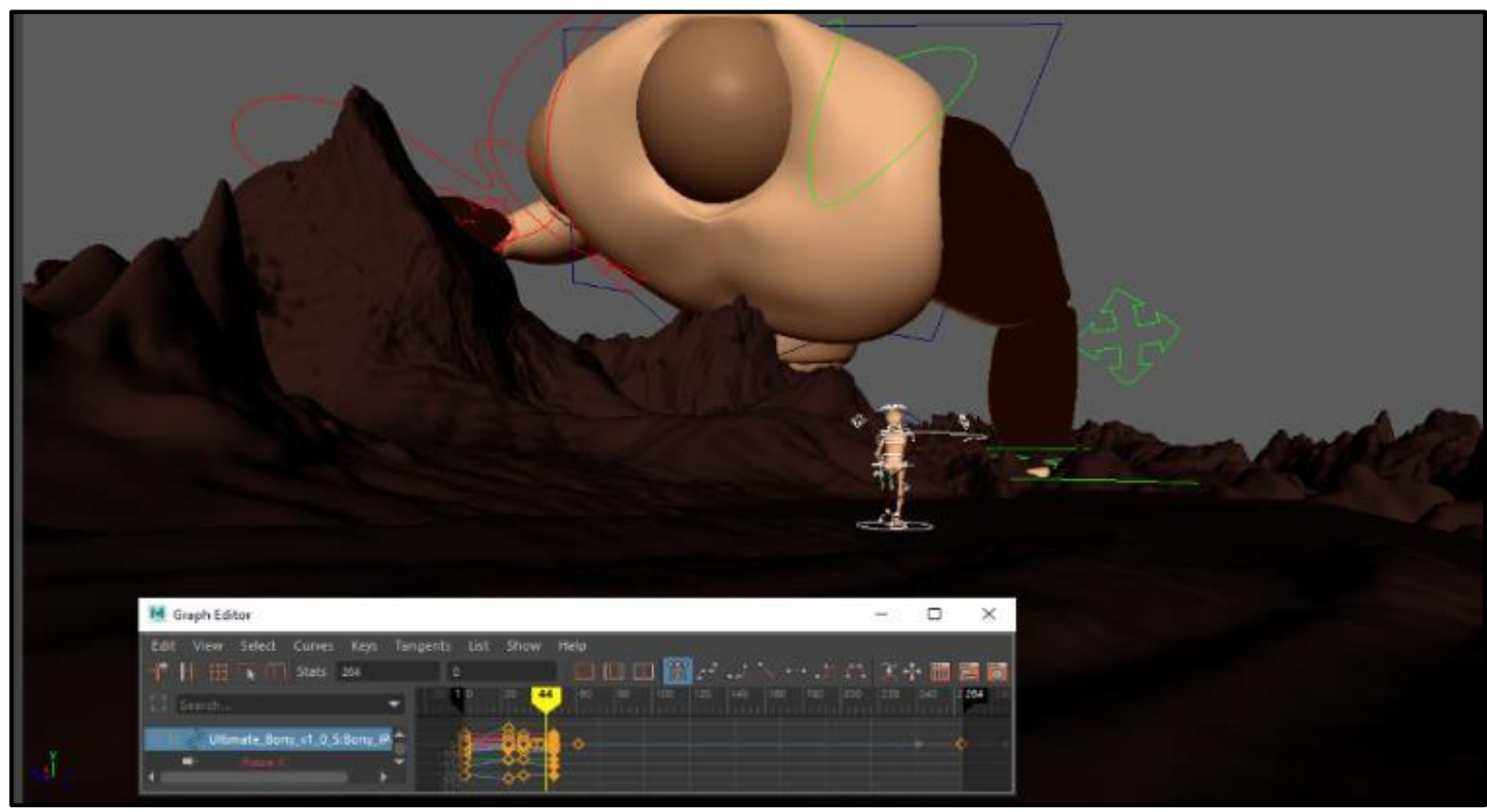

Figure 4. Adding animation keyframes.

Once everything is in place, the rigged model will be animated according to the animatic, and it will sync with the timing and layout like in Figure 4. Next, adding the keyframe for each controller for each model, the time graph panel helps smoothen and create believable motion with weight and purpose. Soon after the rough animation is complete, the scene is improved by adding unique attributes such as horizontal and vertical film aperture to create dynamic scenes that are difficult to view or imagine without the help of the software. Moreover, the additional attributes help to improve the immersion and mood of the scenes.

The dynamic camera angle is one of the challenging aspects to capture when recording live-action footage. The objective is to explore an alternative way to develop this dynamic scene using $3 \mathrm{D}$ playblast as potential references to rotoscope due to its lesser limitation, editable scenario, safer, and arguably lower cost. Hence, the selected scene can be significantly improved with dynamic design elements, and the creative camera works with lesser constraints than in real life. Other than camera shots, placement of the characters in a scene is also essential; for examples, leaving empty spaces around the character's movement to avoid the the feeling of claustrophobic (Heiderich, 2012). Then, the scenes were exported individually according to the subject because clarification is vital during the rotoscope procedure. The sequence will be exported into a PNG sequence on 24 frames per second. Based on the arrangement, a critical keyframe is selected and arrange accordingly to the animatic. The factor of choosing the keyframe consists of cycle animation, static animation, and the beginning and ending for each movement. Finally, keyframe was imported to Adobe Animate CC and Toon Boom Harmony for rotoscoping.

\section{ROTOSCOPING PLAYBLAST FROM 3D MODELS}

\section{a) Adobe Animate CC: Semi Puppet Rotoscope Technique}

Adobe Animate CC, formerly known as Adobe Flash, is an vector-based animation. Its simplicity and lightness made it a popular choice for the online animation industry. The product provides a complete set of animation features and tools for amateurs and professionals. This software can produce various animated projects from Flash-based games, kinetic typography, animation, and any sequence of moving images (Pav, 2021). For this study, the approach of rotoscoping the $3 \mathrm{D}$ playblast for Adobe Animate $\mathrm{CC}$ will be a semi puppet animation method. 


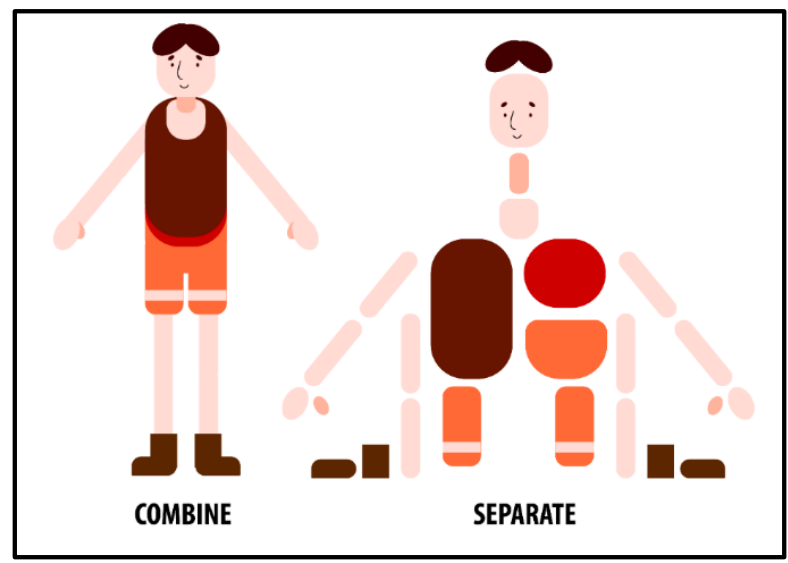

Figure 5. Typical flat 2D animation rig.

The Semi puppet technique is inspired by Adobe After Effect's Puppet Pin Tool. This tool is used to insert and move to deform pins, while Puppet Overlap Tool is used to designate which parts of an image should appear ahead of others when distortion causes parts of the image to overlap (Adobe, 2021). Moreover, several studies have explored its advantages and limitations when using puppeteer characters (Adi Satria, 2019; Eriya \& Zhafiri, 2017). The rig will often look like Figure 5 and can only be used at one limited angle. However, this research's semi puppet method will cover motions in perspective rather than the usual flat angle.

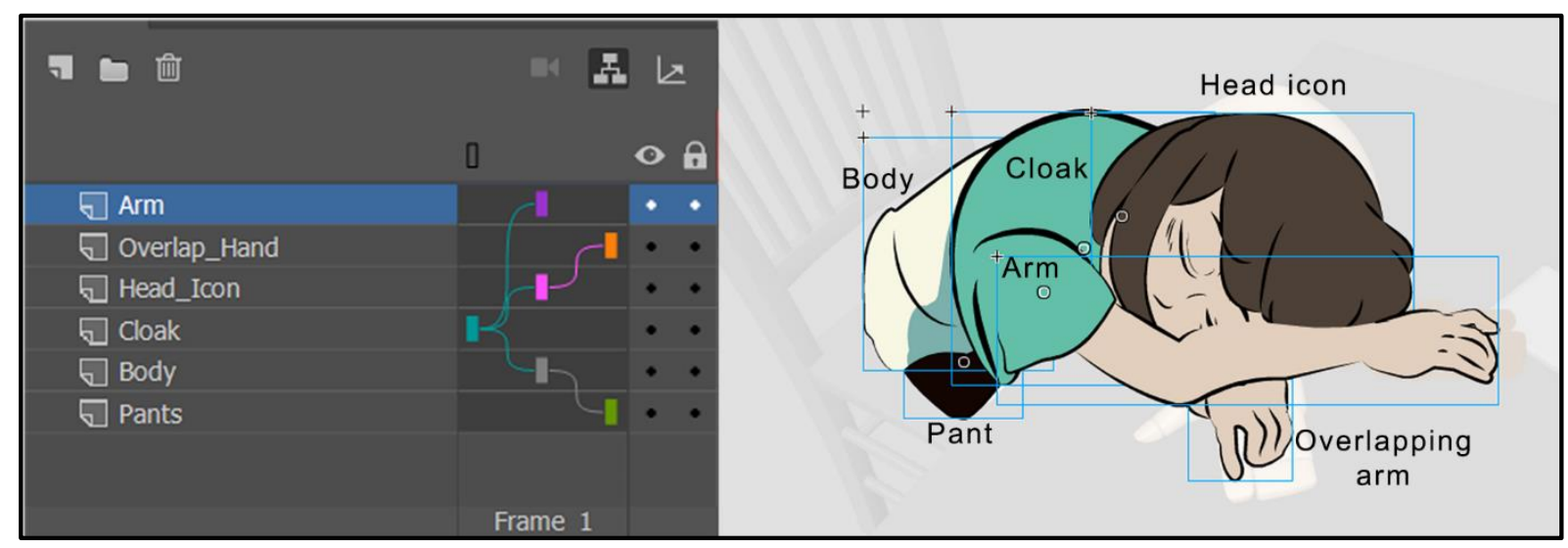

Figure 6. Puppet's rig for perspective animation.

The first step is to export the PNG sequence from Maya and import it inside a new project in Adobe Animate CC's timeline for rotoscoping. Based on the 3D playblast, the composition is adjusted according to the 24 frames per second and the sequence duration. In short, the 3D playblast acts as the reference while rotoscoping the animation using a similar approach to the puppet pin method. Figure 6 shows a high angle shot, showing more depth and perspective of the character. The character is divided into separate significant sections and converted into graphic symbols. While the symbols overlap, each symbol contains each pivoting pin and pinning in its anatomy correct area. Each pin can be used as the anchor when rotating and deforming the symbol. The animation process is like Adobe After Effect's Puppet Pin Tool by keyframing each movement and automatically tweening between the frames.

Firstly, the graphic symbol needs to be arranged in a "parent-child hierarchy system" led by the body as the main symbol (Master control). When the body moves, the entirety of the graphics hierarchy rig that parent to the master control will also move. This includes manipulating the asset warp tool. Keyframes on the master control will influence the entire rig. Additionally, with the versatile feature provided in the program, the symbols can easily replace and re-parent any individual part at any time during the process. Like in 3D animation software, the rig created in Adobe Animate CC can be reused in any scene. Hence, it allows rotoscoping while having a tweening option.

Next, keyframes are added based on the 3D playblast as the reference; each keyframe was automatically motiontweened to move according to the first and last keyframes. In short, tweening generates midway frames between two keyframes or images to give believable movement that progresses smoothly toward the second image. The movement will be refined by manipulating the speed and velocity by using the ease from the tweening feature. Last and not least, adding the background that was created from Adobe Photoshop CC. The background was intentionally 
in grayscale to overlay it by colours layers, and the base colour is enhanced by using custom brushes such as texture brush, pastel brush, dry brush, and solid brush.

\section{b) Toon Boom Harmony: Frame by Frame Rotoscope Technique}

Toon Boom Harmony is a leading 2D animation software that provides the latest sensitivity brushes with a complete professional animation set. One of the attractive features of the software is its flexible and functional tools that allow pressure sensitivity attributes. The software also includes unique features such as Weighted Deform Node for smoothening texture, independent panel for lighting and shading, advanced colour management for environment and effects and a guide for snapping drawing easier. Furthermore, Cartoon Network, Toei Animation, South Park Studio, Disney, and Nickelodeon primarily used Toon Boom Harmony as their standards for animation.

Rotoscoping approach via Toon Boom Harmony is similar to the classical frame-by-frame rotoscoping approach. The brushes and sensitivity of the pencil tool are chosen as the primary tool for the rotoscope. The method was straightforward without any unique technique and technical issue other than spending a considerable amount of time doing the manual drawing for each frame. The process started with importing a separate sequence to individual layers within the timeline for animation rendered from Autodesk Maya as the reference. The process of importing the separated sequence was direct for Toon Boom Harmony, unlike Adobe Animate CC that required specific navigation to import the sequence into the correct timeline layer. However, the Toon Boom Harmony software is dense with various tools with the same objective but different effects when applied in a certain way. Resulting, the increased level of difficulty to master this software compared to the simple yet straightforward approach for Adobe's software.

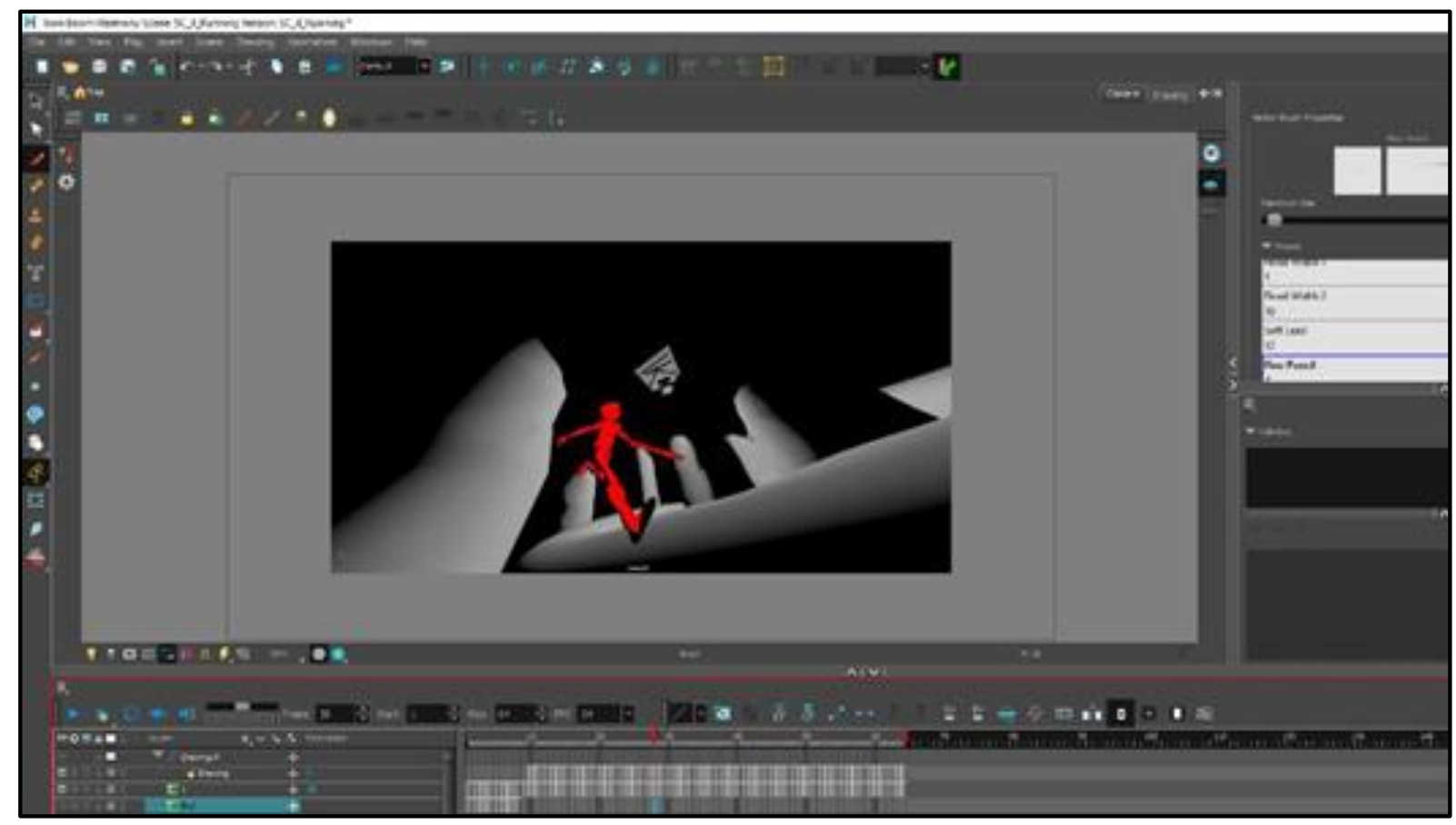

Figure 7. Rotoscoping individual frames.

Once the 3D playblast sequence was placed correctly within the timeline, the next step would be to draw on top of the sequence with a new layer. Generally, the rotoscope is very direct, unlike the Adobe Animate CC that required some moderate planning for the puppet rig. Figure 7, for instance, shows a run cycle animation from an extremely low and distorted angle. Without the 3D playblast as the reference, recreating this scene in real life would be considered a more complicated and higher cost to set up correctly. Unlike actual footage, 3D playblast is editable and convenient to implement and discard material within the virtual environment. Additionally, since the technique is more towards traditional medium, the brush provided in Toon Boom Harmony perform similarly to real traditional media. The software provides brush styles from pastel brush to charcoal, and even Chinese ink brush is made available within the vector brush property panel. Each brush has its customisable slider, such as the pen tilt sensitivity pressure, transparency, smoothing, and even texturise paper. However, the tools selected for the rotoscope are mainly the pencil tool with a 'grunge texture' for the outline. Moreover, a drawing tablet featuring a pressure sensitivity display such as Wacom can help create a better stroke and depth for the linework. As a result, it is essential to acquire a high-quality render vector brush to replicate the aesthetic value of traditional media. The number of keyframes for each scene relies on the complexity of the animation. This scene in Figure 7 uses 24 frames per second, but the rotoscope only took nine frames with ten cycles to moderately animate it due to the simple 
looping motion. Kitahata and Sakamoto (2018) verify that the modification of the frame size, the field of view, and the projection method will minimise the three-dimensional form in the 3D animation, creating the animation more $2 \mathrm{D}$ even though the assets are in 3D. By using this approach, the researchers were able to focus on completing each frame with fewer details.

\section{FINDINGS}

To rotoscope using 3D playblast, the 3D software must establish the assets (models) in the scenes. This includes characters and backgrounds modelled around simple shapes and focusing on silhouettes rather than details. Furthermore, once the assets are completed and animated, additional 3D features such as camera shots can be improved and distort without any collision from other models. Special effects and filters can be added early in the pre-production stages to pre-visualise the scenes further before finalising them in 2D. The next rotoscoping phase is where the assets are separated by PNG sequences and traced over separately. Rotoscoping obvious shapes help determine the proportions and maintain consistency of the overall shapes of the character. The following step is to convert everything back together for the finalised pre-production stages.

In terms of rotoscoping software, Table 2 shows nine aspects that differentiate these two software. First and foremost, the Adobe Animate CC interface is designed for beginners with intuitive navigation. It is not complicated to start any animation. However, its feature is limited and requires other Adobe software or third-party plug-in to complete the animation fully, especially in its post-production stage. Furthermore, the learning curve for this software is low and achievable without worrying about the technical aspect too much. Additionally, easy access to tutorials from online platforms is provided officially or created by experience artists significantly helps new users to learn faster and speed up the productivity within a project. Even so, due to its simplistic design and feature, the rotoscope is limited to simple motion and requires more effort and time to create something more dynamic.

Referring to Table 2, the introduction for its user interface in Toon Boom Harmony is rather intimidating due to its complete set of animation tools that consist of almost all the potential creative processes that the software can give. It features traditional animation to computerised suggestions, a flexible bone rig, and even customisable special effects with a virtual 3D camera. However, unlike the Adobe Animate CC, the learning curve for this software is very high. Due to limited forums, tutorials, discussion, and community participation, it is challenging to acquire specific knowledge to do a specific task. Moreover, this software's actual strength lies in its adaptability to maintain a classical aesthetic without sacrificing modern features and low computing power requirement. While Adobe Animate $\mathrm{CC}$ excels in simple navigation and design, Toon Boom Harmony provides a seamless workplace for traditional animation. The strokes for each brush or pen tool using the tablets are accurate and smooth. The texture and customisable vector brush created a dynamic outline, and the manipulative vector's point exposed the more advanced animation process. Seeing the strength of Toon Boom Harmony, the frame-by-frame rotoscoping method is accomplished despite having minor setbacks during the process.

Table 2. Comparison between two software based on key aspects.

\begin{tabular}{|c|c|c|}
\hline ADOBE ANIMATE CC & ASPECTS & TOON BOOM HARMONY \\
\hline $\begin{array}{l}\text { User-friendly interface to easily navigate and } \\
\text { self-explanatory design. }\end{array}$ & Interface & $\begin{array}{l}\text { Cramped interface but complete animation } \\
\text { package for professional standards. }\end{array}$ \\
\hline $\begin{array}{l}\text { Simplistic feature for complicated non- } \\
\text { animation. Provide more intuitive navigation } \\
\text { and more automation process. }\end{array}$ & Features & $\begin{array}{l}\text { Cramped with specific features and tools } \\
\text { are designed to be used in specific ways. It } \\
\text { might not be intuitive, but it provides more } \\
\text { advanced animation. }\end{array}$ \\
\hline $\begin{array}{l}\text { Easy and straight to the point procedure and } \\
\text { heavily designed for beginners and hobbyists. } \\
\text { However, it required different software to } \\
\text { utilise fully. }\end{array}$ & Learning Curve & $\begin{array}{l}\text { High difficulty learning due to professional } \\
\text { standard without third party plug-in. }\end{array}$ \\
\hline $\begin{array}{l}\text { Countless online tutorials from an } \\
\text { independent artist and straight the point } \\
\text { answer and processes. }\end{array}$ & Online Tutorials & $\begin{array}{l}\text { Little explanations } \\
\text { about the technical aspect and procedure. }\end{array}$ \\
\hline $\begin{array}{l}\text { 3Ghz or faster processor and } 4 \mathrm{~GB} \text { RAM or } \\
\text { more. Adapt with an underperformed } \\
\text { computer in exchange with } \\
\text { slower loading. }\end{array}$ & $\begin{array}{l}\text { Minimum Computing } \\
\text { Requirement }\end{array}$ & $\begin{array}{l}2 \mathrm{GHz} \text { or faster processor and minimum } \\
2 \mathrm{~GB} \text { RAM or more. The apparent difficulty } \\
\text { for compatibility for underperformed } \\
\text { computers. }\end{array}$ \\
\hline $\begin{array}{l}\text { Fast result but limited only to simple } \\
\text { composite. Required time to learn and used } \\
\text { other software to complete. }\end{array}$ & Productivity & $\begin{array}{l}\text { A higher learning curve at the beginning } \\
\text { but gradually increase productivity for the } \\
\text { entire animation. }\end{array}$ \\
\hline $\begin{array}{l}\text { The software specialises in AI-generated } \\
\text { motion, and it is plausible to rotoscope }\end{array}$ & $\begin{array}{l}\text { Rotoscope } \\
\text { Technique }\end{array}$ & $\begin{array}{l}\text { Classical rotoscoping (frame by frame) is } \\
\text { possible with the closest tool to a replicated }\end{array}$ \\
\hline
\end{tabular}




\begin{tabular}{|c|c|c|}
\hline $\begin{array}{l}\text { motion using puppets with well organised } \\
\text { overlapping shapes or graphic symbols. }\end{array}$ & Using playblast & $\begin{array}{l}\text { traditional medium, but its editable feature } \\
\text { could change specific characteristics to fit } \\
\text { the author personally. }\end{array}$ \\
\hline $\begin{array}{l}\text { Semi puppet animation/ Puppet pin } \\
\text { - } \quad \text { Rotoscoping using ai generate motion } \\
\text { lessen the workload of the overall scene. } \\
\text { - Reusable character assets that are only } \\
\text { required to build once. } \\
\text { Assets are editable and have supporting } \\
\text { library save } \\
\text { The timing and speed of the character's } \\
\text { motion are more flexible to edit and } \\
\text { improve. } \\
\text { Creative ways to find plausible shortcuts } \\
\text { with only motion without the need for a } \\
\text { new keyframe }\end{array}$ & $\begin{array}{l}\text { Rotoscope Technique } \\
\text { Advantages }\end{array}$ & $\begin{array}{l}\text { Classical Rotoscope (Frame by Frame) } \\
\text { - } \quad \text { Closest tool to aesthetically maintain } \\
\text { traditional animation } \\
\text { Keyframe characteristics such as } \\
\text { outline can be changed without the } \\
\text { need to start over a new sequence. } \\
\text { Everything can be selected as a whole } \\
\text { and change at the same time. } \\
\text { Colours can be auto-generated from } \\
\text { the previous keyframe if the next } \\
\text { keyframe is almost identical. } \\
\text { Potentially have the most freedom in } \\
\text { terms of stylise drawing. }\end{array}$ \\
\hline $\begin{array}{l}\text { Technical and organising would require } \\
\text { more focus due to assets are loaded } \\
\text { according to the Adobe library. } \\
\text { Assets are limited to its motion } \\
\text { constraint } \\
\text { - Library could be messy and hectic when } \\
\text { dealing with hundreds of assets and its } \\
\text { smaller shapes within the library } \\
\text { Motion sometimes feel and look } \\
\text { artificial with ai generated motion }\end{array}$ & Disadvantages & $\begin{array}{l}\text { - Intensive workload is expected for } \\
\text { classical rotoscope } \\
\text { Required to have more effort to } \\
\text { maintain consistency } \\
\text { Auto-generate colours are not } \\
\text { plausible to give an accurate filling } \\
\text { when dealing with fast-fast motions } \\
\text { such as fighting. } \\
\text { It is required to connect each line } \\
\text { carefully to make it possible to fill in } \\
\text { colour. } \\
\text { More complex designs required more } \\
\text { workload to stay consistent. }\end{array}$ \\
\hline
\end{tabular}

By comparing each rotoscoping technique in Table 2, both are capable of rotoscoping animation from animated 3D playblast. In summation, the semi puppet tool is helpful for simple rotoscoped motions. It provides various shortcuts that lessen the animator's workload. Meanwhile, classical rotoscoping is achievable with Toon Boom Harmony software while providing more creative design choices, including advantages and disadvantages in the traditional medium. Using 3D playblast has more potential to visualise the animators' expectations with lower cost, and lesser equipment compares with actual footage. However, real footage provides more realistic detail than 3D playblast. Also, 3D playblast would not entirely replace the benefits provided by actual live-action footage.

\section{DISCUSSION}

The study explores and evaluates different rotoscoping technique approaches via two industry-leading software (Adobe Animate CC and Toon Boom Harmony). This section will discuss the overall aspects such as the production speed, quality animation, technical aspect, and learning difficulty. Moreover, it also covers different advantages and disadvantages when using 3D playblast for rotoscoping animation.

First, Adobe Animate CC design and approach suit beginners and professionals due to its simplicity and straight point feature. However, Adobe Animate is difficult to embrace fully the feature of what makes an animation creative. It limits the user's creative process with its simplicity feature and forced to rely on third-party plug-ins or builds to create customisable tools to make the software more versatile and adaptable for production stages. For instance, Adobe Animate CC have two main tools to develop linework, a pencil tool and a brush tool. However, the small pool of readymade brushes and its current customisable are limited with only the shape and pressure. Moreover, brush tools and paintbrush tools are not selected to do rotoscoping. This is because it is unsuitable for outline due to its bristle strokes and uncontrollable ink pressure created more mess and effort to complete a single stroke. The technical challenge that can potentially occur within the rigging process is relatively rare during the production stage. However, it messed up the library arrangement and must restart to square one for the puppet rig. The software heavily depends on the performance of the current computer, with higher specifications resulting in better performance to automatically create an in-between keyframe. As a result, lower-spec acquired find ways to develop additional customisation because the program is not designed to adapt with lower computing power. Although rotoscoping is not the best option for Adobe Animate CC, the puppet rig method is more versatile and simpler to complete. With its parent-child hierarchy and tweening attributes, the program can create a powerful combination of frame-by-frame animation and puppet animation. Hence, a hybrid of the mixed rotoscoping technique should be utilised.

The second software is Toon Boom Harmony. The software is known for its more significant initial learning curve 
due to the feature, and tools are designed only for niche uses. Comparing to Adobe Animate CC easier to use brushes, Toon Boom Harmony provide a more feature-rich and high-quality texture brush, including bitmap drawing and painting tool mode with no computing problem despite having the same underperformed computer. Furthermore, the composition is available within the software itself by providing robust compositing tools and compositing systems. Meanwhile, Adobe Animate CC relies on other software within the Adobe suite like Adobe After Effect to integrate visual effects. The same factor occurs as well about rigging tools. Instead of a simple animate bone puppet provided in Adobe Animate CC, Toon Boom Harmony adds advanced features to the puppet to create more flexible and adaptable movement. This software's most substantial aspect is its imitation of brush that replicate texture from traditional media. Hence, classical rotoscoping within Toon Boom Harmony accomplishes similar objectives as traditionally rotoscoping on paper.

There is a noticeable difference between the use of rotoscope using film footage with 3D playblast animation, specifically, the limitation and the sense of realism between pre-visualisation and execution. Rotoscoping actual footage has evolved from classical rotoscoping individual frames to specialise software that automatically detects algorithms to separate subjects from each other. Moreover, the advantage of using actual footage maintains the most vital aspect of realism. For example, details such as the casting of shadow and light, and believability of movements are preserved. On the other hand, it would be difficult to exaggerate a particular aspect of the animation. This includes the aspect of dynamic camera shots that require advanced camera technology to pull off. Despite its advantages, rotoscoping from film footage requires more planning and workload when rotoscoping frame-by-frame. Although AI algorithm is available to detect subject motion in modern software, sooner and later, there will be a scene that is difficult to detect with cluttered background or merged subjects.

Rotoscoping from 3D playblast animation is still a new concept. There is no correct conceptual way to provide previsualised 3D animation for rotoscoping. However, 3D playblast animation can surpass the limitation of actual footage. For instance, since everything is computer-generated, creating models and scenarios is limited to the animator's imagination. A creative shot can improve the audience's experience because it stood out from the scenes and became a memorial plot point. Despite having limitless potential, rotoscoping using 3D playblast animation sacrifices a few aspects of realism in providing more creative freedom. The exploration concludes that both classical rotoscope and puppet animation are effective. With an option to export the subjects separately from each other, it is already a significant factor when rotoscoping using 3D playblast animation.

The assets to pre-visualise the scene require some time to establish, especially background and character should be roughly made for rotoscoping purposes. As a result, there are several suggestions to be considered when applying 3D playblast animation for rotoscope. First, when creating or selecting characters to be rotoscope, it is vital to focus only on the fundamental basic shapes when building the model. It is not recommended to be animated on a full detail rig for 3D playblast. It would hinder the overlapping shape from its details and acquire more time to animate every characteristic fully. The purpose of playblast is to create a quick alternative to provide grounding and using it as a reference when animating complex shots, maintaining the body proportion and being able to help identify the shape of the motion at a dynamic angle. This method is also similar to animating background, where shapes and silhouettes are the priorities.

Furthermore, once the model and the rough animation is complete, the scene should be exported separately according to its purpose and avoiding merging everything into a single sequence. For instance, characters, background, and even secondary special effects should be converted into PNG sequences separately to be rotoscoped. This would not just help to adjust the compositing more easily. It is also helpful to create a safe environment when adding and discarding elements without destroying the subject surrounding them. It is recommended to identify the crucial keyframe from the sequence rather than trace every individual frame. Some frames are often similar within those 24 frames, and they should not be recognisable at first glance. By focusing on the critical keyframe, in-between can now be chosen to create a somewhat believable scene without rotoscoping every individual frame. For instance, this animation project was animated in 13 frames per second, but the playblast animation was exported on 24 frames per second; a higher frame rate will be added if a high pace scene requires more frames to be animated.

In summary, both software (Adobe Animate CC \& Toon Boom Harmony) is plausible for rotoscoped animation. Adobe Animate CC is simple and well-designed in terms of navigation, more accessible to implemented auto inbetween for animation and excellent for puppet pin animation for a project that focuses only on AI assistance when animating. It also provides a versatile animation style, switching from puppet pin rigging to manually drawn animation within the same layers. Hence, the animation method can be considered semi puppet animation due to its hybrid nature switching from puppet and independent frame. On the other hand, Toon Boom Harmony is the closest form of digitising traditional medium software that has a traditional aesthetic feature on its brushes. The software may be intimidating at first, and some features are hidden under specific navigation. Still, once users have familiarised themselves with the software, the production would be significantly faster, and shortcut keys will 
eventually be used. Both can be used for rotoscope, and 3D playblast animation has the potential to grow as a concept in producing high-quality scenes. In addition, the advantage of using 3D playblast is to empower animators to create more creative and dynamic shots. Additionally, it is recommended to render each model separately within the 3D playblast on its sequence and emphasise the overall shapes (silhouette) rather than details. As a result, 3D playblast can provide further guidance for animators to develop 2D animation via rotoscoping, improving their overall shot. This approach is also beneficial in experimental educational animation research. For example, comparison studies from various 2D animation, 3D animation, and live-action videos can be conducted by incorporating the rotoscoping techniques to create 2D animation samples based on 3D models or forms (Lucas, 2019).

Nevertheless, there are limitations to this study. Researching 3D playblast into 2D animation rotoscoping technique is quite a niche area. Hence, there are limited studies published focusing on the topic. Moreover, when an animation uses rotoscope techniques, it would be difficult to identify the scene by only observations without acquiring indepth information of the development process. Furthermore, during the exploration, the researchers could not cover in-depth all the advanced mechanics of the software. Instead, this study focuses on the unique aspects of the animation software by incorporating 3D playblast animation as a reference for rotoscoping dynamic scenes.

\section{CONCLUSION}

This research explores alternative rotoscoping techniques such as using 3D playblast to create a more refined 2D animation. Two different rotoscoping approaches were explored to achieve the objectives and analysed to develop a proper dynamic scene. The workflow from rotoscoping using 3D playblast into $2 \mathrm{D}$ animation shows that it is achievable to create a dynamic animation scene using 3D models. Accordingly, using 3D playblast as a viable alternative to visualise motion can help animation studios improve their rotoscope composition quality and create creative ways to convey complex but crucial scenes in the story.

\section{ACKNOWLEDGMENTS}

We would like to thank our animation lecturers, classmates, and family members for the continuous support and encouragement throughout the completion of this research and animation project during the pandemic.

\section{REFERENCES}

A-ha. (2010, January 7). A-ha - Take On Me (Official Video) [Remastered in 4K]. Retrieved September 15, 2021, from https://www.youtube.com/watch?v=djV11Xbc914

Adi Satria, D. (2019). Analisis Penggunaan Puppet Pin Rigging Pada Pergerakan Karakter Animasi 2D. Ejournal.Upbatam.Ac.Id, (0274). Retrieved from http://ejournal.upbatam.ac.id/index.php/jif/article/view/1346

Adobe. (2021, May 16). Animating with Puppet tools in After Effects. Retrieved September 14, 2021, from https://helpx.adobe.com/in/after-effects/using/animating-puppet-tools.html

Agarwala, A., Hertzmann, A., Salesin, D. H., \& Seitz, S. M. (2004). Keyframe-based tracking for rotoscoping and animation. ACM SIGGRAPH 2004 Papers, SIGGRAPH 2004, 1(212), 584-591.

Blake, A., \& Isard, M. (1998). CONDENSATION - Conditional Density Propagation for Visual Tracking. International Journal of Computer Vision, 29(1), 5-28. Retrieved from http://www.springerlink.com/index/xl887466h454318k.pdf

Bratt, B. (2011). Rotoscope Technique and tools for the Aspiring Artist (Vol. 148).

Callum May, \& Kim Morrissy. (2018, January 11). Interview: Yuichi Terao, Chief of Ufotable's Digital Team Anime News Network:SEA. Retrieved September 12, 2021, from https://www.animenewsnetwork.com/feature/2018-01-10/interview-yuichi-terao-chief-of-ufotable-digitalteam/.125658

Coulthard, P. (2020, April 17). Blender Grease Pencil - Storyboard Animatic Tutorial - 6 of 6 - Rotoscoping in 3D space, import FBX. Retrieved September 16, 2021, from https://www.youtube.com/watch?v=D9_kXQFeQ2w

Eriya, \& Zhafiri, F. A. (2017). Pembuatan Film Animasi "TEMAN" Menggunakan Teknik Puppet Pin dan Duik sebagai Media Edukasi Anak. Multinetics, 3(1), 32.

Gabe, M. (2014). CAMERA ANGLES: the Art of Manipulation. Retrieved from http://www.elementsofcinema.com/cinematography/camera-angles-and-composition/

Gossman, R. (2012). The Animation Production Process, 1-8.

Heiderich, T. (2012). Cinematography Techniques: The Different Types of Shots in Film. Videomaker, 1-17.

Irani, M. (2002). Multi-frame correspondence estimation using subspace constraints. International Journal of Computer Vision, 48(3), 173-194. 
Jaran, S. (2015). History Of The Rotoscoping Technique - Brattle Theatre Film Notes. Retrieved September 11, 2021, from https://www.brattleblog.brattlefilm.org/2015/09/11/history-of-the-rotoscoping-technique-2$3231 /$

Johnson, P. (2014, June 13). Rotoscoping over 3D reference. Retrieved September 15, 2021, from https://www.youtube.com/watch?v=X0taAlZ_MxA

Johnson, P. (2018, November 10). How To Animate an 80s Anime Ship. Retrieved September 15, 2021, from https://www.youtube.com/watch?v=MMUcafde5A4\&t=0s

Karpathyova, I. (2017). Rotoscoping Design for Bodily Technique and Interdisciplinary Research on Animation as Embodied Practice. OCAD University, 4, 9-15.

Kitahata, K., \& Sakamoto, Y. (2018). Evaluation of reducing three-dimensionality of movement to create 3DCG Animation looks more like 2D Animation. SIGGRAPH Asia 2018 Posters, SA 2018, 1, 2017-2018.

Lucas, T. (2019). Exploring the effect of realism at the cognitive stage of complex motor skill learning. $E$ Learning and Digital Media, 16(4), 242-266. https://doi.org/10.1177/2042753019835893

Pav, N. (2021, March 21). Adobe Animate Review: Best for Hobbyists or Creative Pros? Retrieved September 14, 2021, from https://www.softwarehow.com/adobe-animate-review/

Riverman Media. (n.d.). From 2D to 3D and Back: Rotoscoping in Pizza Vs. Skeletons. Retrieved from https://rivermanmedia.com/from-2d-to-3d-and-back-rotoscoping-in-pizza-vs-skeletons/

Ruddell, C. (2012). "Don't box me in": Blurred lines in waking life and a Scanner Darkly. Animation, 7(1), 7 23.

Sabiston, B. (2017, June 1). Waking Life: Rotoscoping (HOW THE MOVIE WAS MADE). Retrieved September 15, 2021, from https://www.youtube.com/watch?v=UJwjSV6VEpc

Spot Ceres. (2012, April 16). SPOT CERES - LA CITTÀ HA BISOGNO DI EROI (spot 2012). Retrieved September 15, 2021, from https://www.youtube.com/watch?v=e9Zm7AqA0G4

Swarbrick, R. (2021, April 30). Rotoscope: The dark art of animation | shots. Retrieved September 15, 2021, from https://shots.net/news/view/rotoscope-the-dark-art-of-animation

Team Miracles. (2020, March 17). Rotoscoping Over 3D Animation with Grease Pencil | Blender 2.81. Retrieved September 15, 2021, from https://www.youtube.com/watch?v=o6w-oWr-AvA

Torresani, L., \& Bregler, C. (2002). Space-Time Tracking. Lecture Notes in Computer Science (Including Subseries Lecture Notes in Artificial Intelligence and Lecture Notes in Bioinformatics), 2350, 801-812.

Ufotable. (2013). ufotable digitalteam demoreel on Vimeo. Retrieved from https://vimeo.com/77675596

Uğur, U. Y. (2014). Ultimate Rigs for FREE on Behance. Retrieved September 12, 2021, from https://www.behance.net/gallery/17774421/Ultimate-Rigs-for-FREE 